A RADIATION ENERGY-ANGLE ALGORITHM FOR USE IN PERSONNEL DOSIMETRY
Margarete Ehrlich

U.S. DEPARTMENT OF COMMERCE Natlonal Instltute of Standards and Tochnology lonizIng Radlation DIvision Galthersburg, MD 20899
U.S. DEPARTMENT OF COMMERCE Robert A. Mosbacher, Secretary MATIONAL INSTITUTE OF STANDARDS AND TECHNOLOGY

John W. Lyons, Director 



\section{A RADIATION ENERGY-ANGLE ALGORITHM FOR USE IN PERSONNEL DOSIMETRY}

\section{Margarete Ehrlich}

U.S. DEPARTMENT OF COMMERCE Natlonal Instlute of standards and Technology Ionlzing Radlation Divislon Galthersburs, MD 20899

Aprll 1990

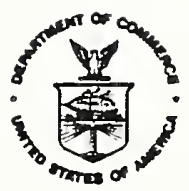

U.S. DEPARTMENT OF COMMERCE Robert A. Mosbacher, Secretary NATIONAL INSTITUTE OF STANDARDS AND TECHNOLOGY

John W. Lyons, Director 



\section{Contents}

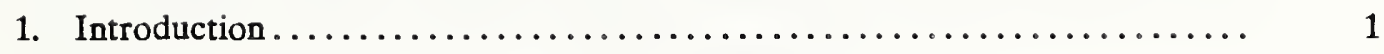

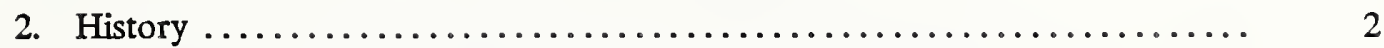

2.1 Dosimeter Calibration for Photon Dosimetry (Historical) ........... 2

2.2 Algorithm for Evaluating $H$ from Measurements on the Field

Dosimeters Irradiated with Photons (Historical) .............. 2

3. Generalization of Historical Method ........................ 3

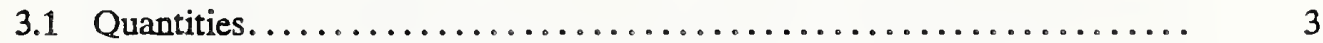

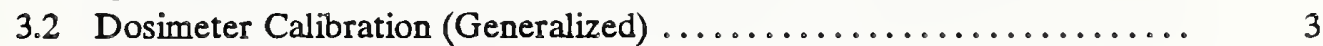

3.3 Algorithm for Evaluating $H$ from Measurements on the Field

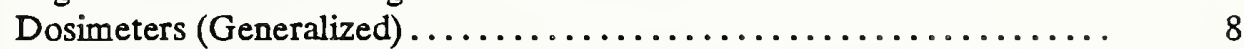

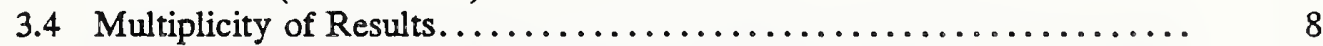

3.5 Assigning Weights to the Resulting Individual $H$ Values............ 8

3.5.1 Mechanics Involved in Arriving at $(E, \theta)$ Values............. 8

3.5.2 Averaging the $H$ Values Along the Curve of Intersection...... 9

4. Accuracy of the Method $\ldots \ldots \ldots \ldots \ldots \ldots \ldots \ldots \ldots \ldots \ldots \ldots \ldots \ldots \ldots \ldots \ldots$

5. Acknowledgments..................................... 10

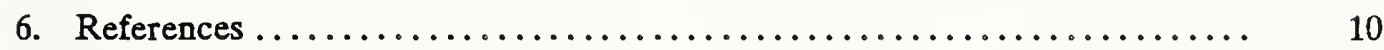

\section{List of Figures}

Figure 1. Example of Three-Dimensional Spline of a Response Function,

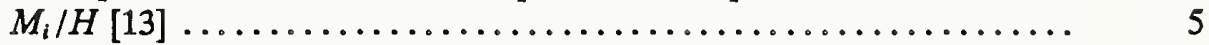

Figure 2. Example of Three-Dimensional Spline of a Response Function,

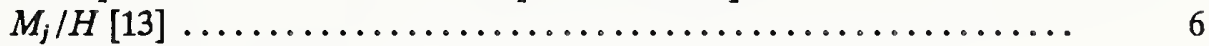

Figure 3. Example of Three-Dimensional Spline of a Response-Function

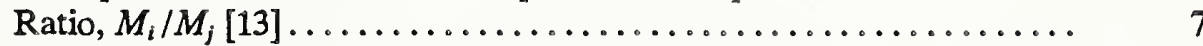





\title{
A Radiation Energy-Angle Algorithm for Use in Personnel Dosimetry
}

\author{
Margarete Ehrlich \\ National Institute of Standards and Technology \\ Gaithersburg, MD 20899
}

\begin{abstract}
In an earlier publication, the author described an algorithm for arriving at the value of the individual dose equivalent from the readings of dosimeters having two areas of different energy- and angle-response functions. The algorithm is briefly reviewed here, and suggestions are made for obtaining, by numerical means, a plausible average of all solutions possible for a given type of dosimeter and number of calibration data.
\end{abstract}

Key words algorithm; angular dependence; energy dependence; individual dose equivalent; personnel dosimetry; plausible average of solutions; response functions; response ratios; surfaces in three dimensions; three-dimensional spline fits.

\section{Introduction}

In the days prior to the existence of the "effective dose equivalent" and the "individual dose equivalent," personnel dosimeters were constructed with only little attention to the angular dependence of their response. Dosimeter calibration was performed as if the dosimeters were to be used solely under perpendicular incidence of both the primary and scattered radiation, and dosimeter evaluation was in terms of quantities that ignored the asymmetries in the absorption of radiation in the human body. This situation changed with the advent of the effective dose equivalent and of some of the new operational dose-equivalent quantities [1], which take these differences into account. As a consequence, more studies are being performed on angular dependence of the response of personnel dosimeters in current use. (See, e.g., Marshall et al. [2], King [3] and the various papers on this topic presented at two seminars on radiation protection quantities for external exposure held in 1985 [4] and 1988 [5].) Also, the revised personnel-dosimetry performance test document, ANSI N-13.11 [6], will go far beyond the old edition [7], which recommended only that resulis of such studies be available.

While new dosimeter construction probably will take angular-dependence requirements into account, in many instances the use of older types of dosimeters probably will continue for some time. This report deals with the evaluation of readings on some of these older types of dosimeters in terms of either air kerma or dose equivalent by a procedure that may make the replacement of these dosimeters altogether unnecessary. A brief review is given of this evaluation procedure ("evaluation algorithm") which was first presented in 1988 at the Seminar on Radiation Protection Quantities mentioned above [8], and steps are suggested for improving it. 


\section{History}

In the early days, photographic emulsions were essentially the only radiation-sensitive elements employed for personnel dosimetry - with personnel dosimeters, in general, incorporating both nuclear-track emulsions for neutron dosimetry and conventional emulsions for photon and beta-particle dosimetry. The response functions of photographic emulsions are known to be strongly dependent on radiation energy and angle of radiation incidence. ${ }^{1}$ Nevertheless, the customary way to interpret response to photons or beta particles in terms of a quantity related to the radiation exposure on an individual was to assume perpendicular radiation incidence upon the front face of the dosimeter. After isolating the effect of neutrons, discrimination between the effects of beta particles and photons of different energies was accomplished by incorporating into each personnel dosimeter a series of different filters or filter combinations, covering most of the surface area of the conventional emulsions, and providing one or two areas of different response to beta particles and photons, and at least two further areas of different response to photons of different energies. (See, e.g., the review given in IAEA Safety Series No. 8. [9]) The resulting evaluation procedure used for photon or beta-particle dosimetry is described below, with contemporary nomenclature.

\subsection{Dosimeter Calibration for Photon Dosimetry (Historical)}

The dosimeters were calibrated solely with radiation of perpendicular incidence. Calibration involved the following steps:

(a) irradiating the dosimeters in known radiation fields covering the entire range of interest of either monoenergetic photons of energy, $E$, or of narrow spectral bands of photons of an average energy, $E$, at known levels of the dosimetric quantity, $H_{,}{ }^{2}$ in terms of which dosimeter readings were to be interpreted;

(b) measuring optical density, $M$, in all emulsion areas of all dosimeters, and plotting or tabulating the values of $M$ as a function of $H$, with $E$ as the parameter.

(c) determining the response functions, $M_{i}(E) / H(E)$, under the $i$ th filter, similarly $M_{j}(E) / H(E)$ under the $j$ th filter, and so on for all filter combinations and all energies, $E$; then taking the ratios, $M_{i}(E) / M_{j}(E)$ etc., of these response functions, and plotting or tabulating them as a function of $E$ for all calibration energies.

\subsection{Algorithm for Evaluating $H$ from Measurements on the Field Dosimeters Irradiated with Protons (Historical)}

Evaluation of $H$ on the field dosimeters involved:

(a) measuring the values of $M_{i}, M_{j}$ etc., under each filter of each field dosimeter, and forming the response ratios, $M_{i} / M_{j}$ etc.;

(b) with the aid of the calibration relationships obtained for the response ratios in step (c) of section 2.1, finding the photon energy (or, in general, energies), $E$, corresponding to each of these ratios;

\footnotetext{
1 "Response function" is the customary term for "response as a function of radiation energy," where "response" is defined as dosimeter reading per unit of the chosen dosimetric quantity.

${ }^{2}$ In this report, the dosimetric quantity is represented by the symbol, $H$, regardless of whether it is the old-time "exposure," air kerma, or one of the operational dose-equivalent quantities.
} 
(c) with the aid of the calibration relationships between $M$ and $H$ obtained in step (b) of section 2.1 with the parameter $E$, finding the values of $H$ corresponding to each measured value of $M_{i}, M_{j}$ etc., for the energies determined in the preceding step.

This algorithm seems more straightforward than it really is, because, in general, both step (b) and the fact that the energy spectrum encountered in the field does not match the one used for the calibration, may result in more than one energy value. The final selection of a value for $H$ therefore necessitates a judicious choice.

In the following, it will be shown how the algorithm described above can be generalized to apply to the current problem of determining the value of the chosen quantity, say the individual $H$, with the angle of radiation incidence, $\theta$, being assumed to vary between at least $0^{\circ}$ and $90^{\circ}$.

\section{Generalization of the Historical Method}

This generalization should be applicable to all types of radiation for which one uses personnel dosimeters that (a) have response functions depending strongly on radiation energy and angle of radiation incidence, and (b) incorporate at least two areas of different energy - and angle-response functions. It should be applicable also to instances in which two types of detectors with different response functions are used either in the same dosimeter or in two dosimeters worn side by side. Although not explicitly treated, this case is implied in all the following procedural steps, in which reference is made to "detectors $i$ and $j$." The method, which does not require separate knowledge of the energy spectrum and the angular distribution of the radiation encountered in the field, was described by the author in a presentation made at the 1988 Radiation Protection Dosimetry Seminar [8]. In this presentation, relatively encouraging results were shown of the author's attempt to apply the algorithm in a rough and unsophisticated manner to one particular dosimeter type in current use. At the same Seminar, a similar idea for an algorithm was touched upon also in a presentation from the Physikalisch-Technische Bundesanstalt [10].

In the following, a brief review is given of the quantities employed, and of the calibration method and the evaluation algorithm. This is followed by suggestions of ways to reduce the uncertainties arising from the multiplicity of the results obtained.

\subsection{Quantities}

Here, the dosimeter indication $M$ applies to any type of radiation effect measured for any type of dosimeter. The quantity $H$ is taken to be the individual dose equivalent (shallow or penetrating) [1], which is still awaiting its final definition [11,12]. Both $M$ and $H$ are taken to be functions of radiation energy, $E$, and of angle of radiation incidence, $\theta$, with $H(E, \theta)$ being known at present at least approximately from computations of the directional dose equivalent. (The quantity $H$ could have been taken also to represent air kerma, and then could have been designated by $H(E)$.)

\subsection{Dosimeter Calibration (Generalized)}

For dosimeters incorporating two detectors, $i$ and $j$, with different response functions, dosimeter calibration entails:

(a) irradiating the dosimeters in known radiation fields of the type of radiation under consideration, covering the entire energy and angle range of interest and known levels of 
the quantity, $H$;

(b) measuring dosimeter indication, $M$, as a function of $H$, for detectors $i$ and $j$, over the range of energies of interest and over the range of angles of radiation incidence between at least $0^{\circ}$ and $90^{\circ}$, and tabulating or plotting the values of $M$ as a function of $H$ for all values of $E$ and $\theta$;

(c) forming the response functions, $M_{i}(E, \theta) / H(E, \theta)$ and $\mathrm{M}_{j}(E, \theta) / H(E, \theta)$, and tabulating or plotting them against both $E$ and $\theta$ for all values of $i, j, E$ and $\theta$; and

(d) forming the response ratios,

$$
\left\{M_{i}(E, \theta) / H(E, \theta)\right\} /\left\{M_{j}(E, \theta) / H(E, \theta)\right\},
$$

and tabulating or plotting them against both $E$ and $\theta$ for all values of $i j, E$ and $\theta$.

The data may be plotted either in two dimensions, resulting in families of curves with either $E$ or $\theta$ as the parameter, or in three dimensions, resulting in a family of surfaces. Several types of numerical fitting procedures, e.g., polynomial least-squares fits or various types of spline fits, are available for use in either case.

Examples of families of curves in two dimensions, for only four energies and three angles, drawn simply by eye, were included in the earlier presentation [8]. For some of the same data, examples of surfaces in three dimensions for $M_{i} / H$ versus $E$ and $\theta$ and for $M_{i} / M_{j}$ versus $E$ and $\theta$ were produced by Boisvert [13] with a computer program on the NIST mainframe computer. These surfaces are shown in figures 1,2 and 3. 


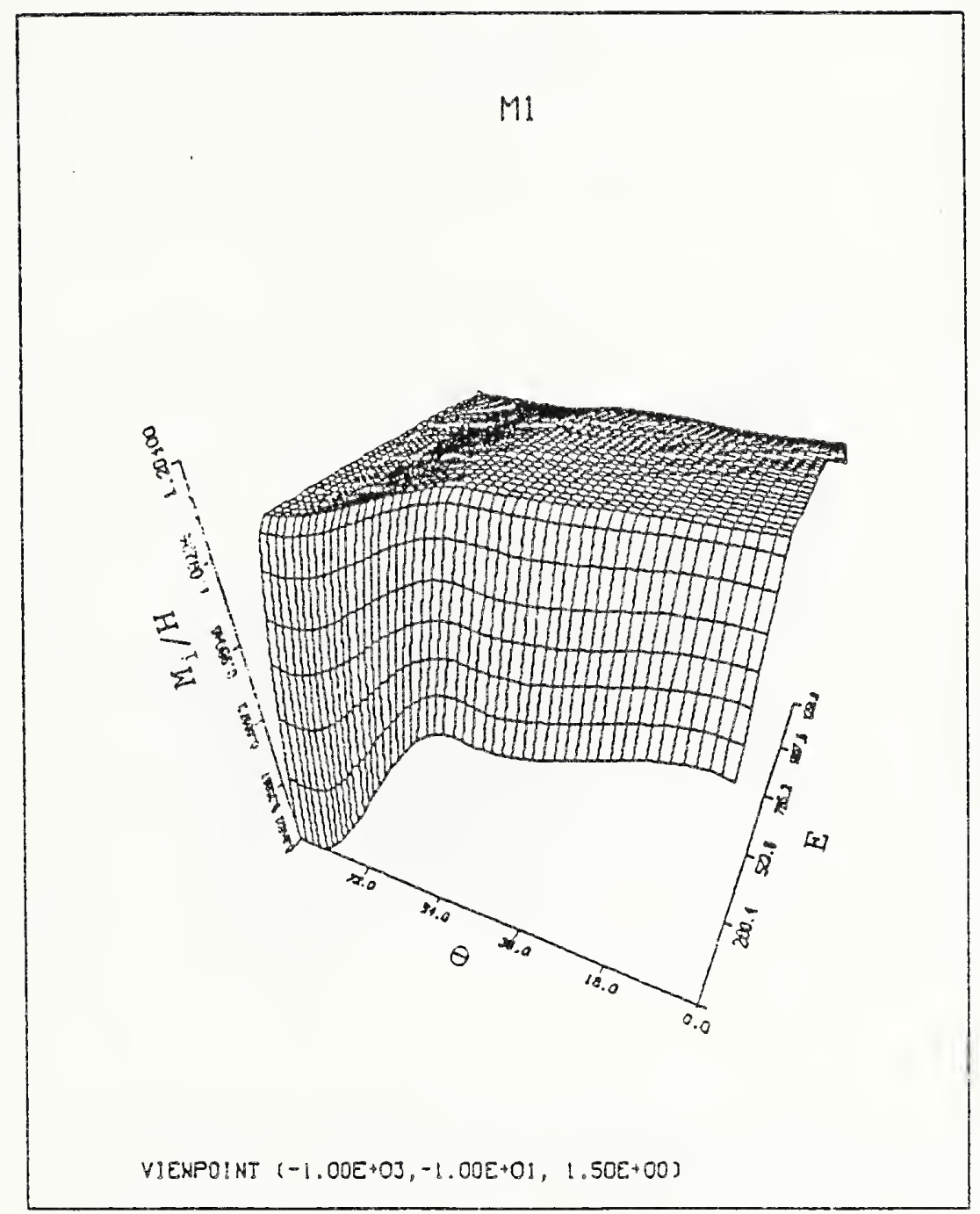

Figure 1. Example of three-dimensional spline of a response function, $M_{l} / H[13]$. 


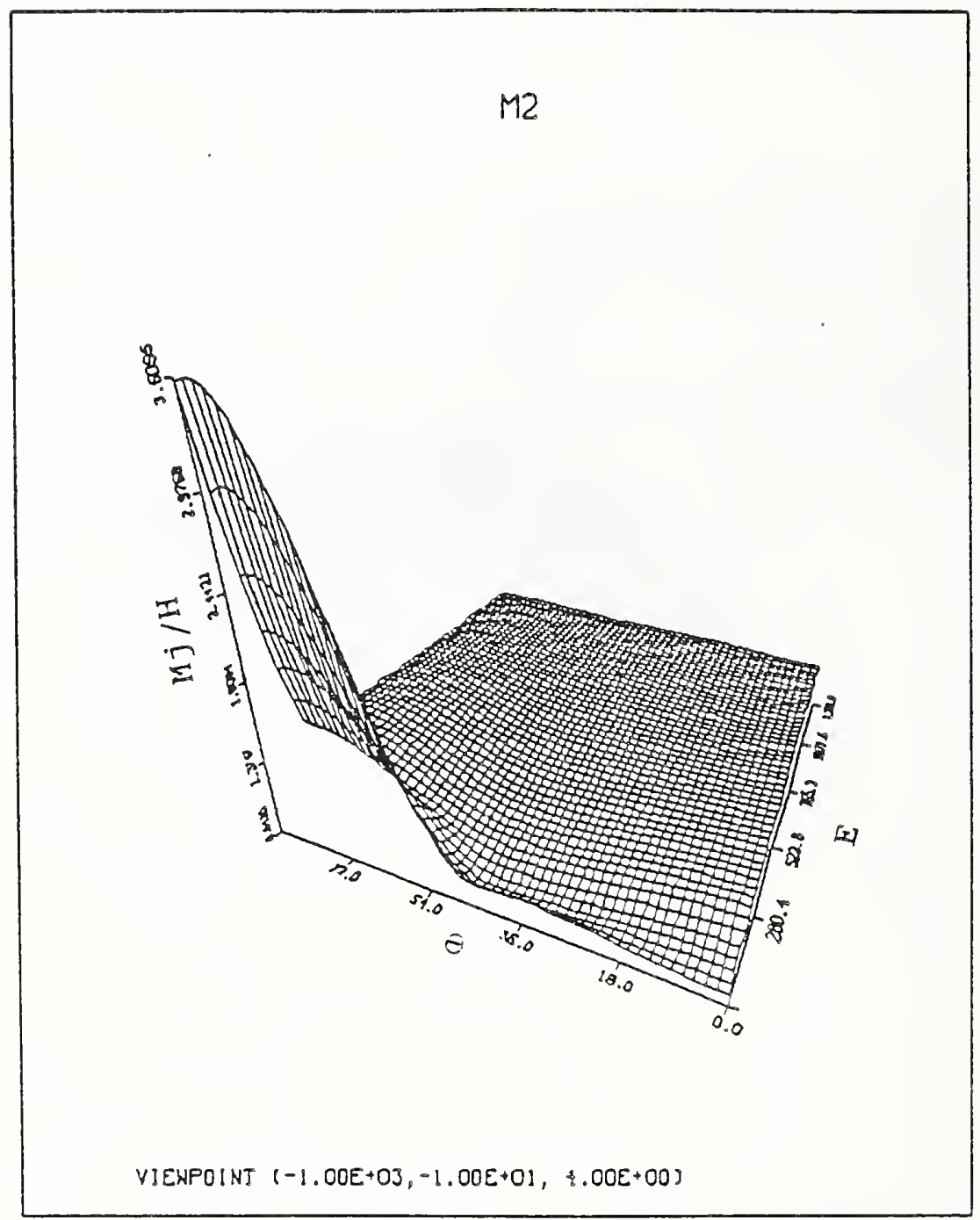

Figure 2. Example of three-dimensional spline of a response function, $M_{j} / H[13]$. 


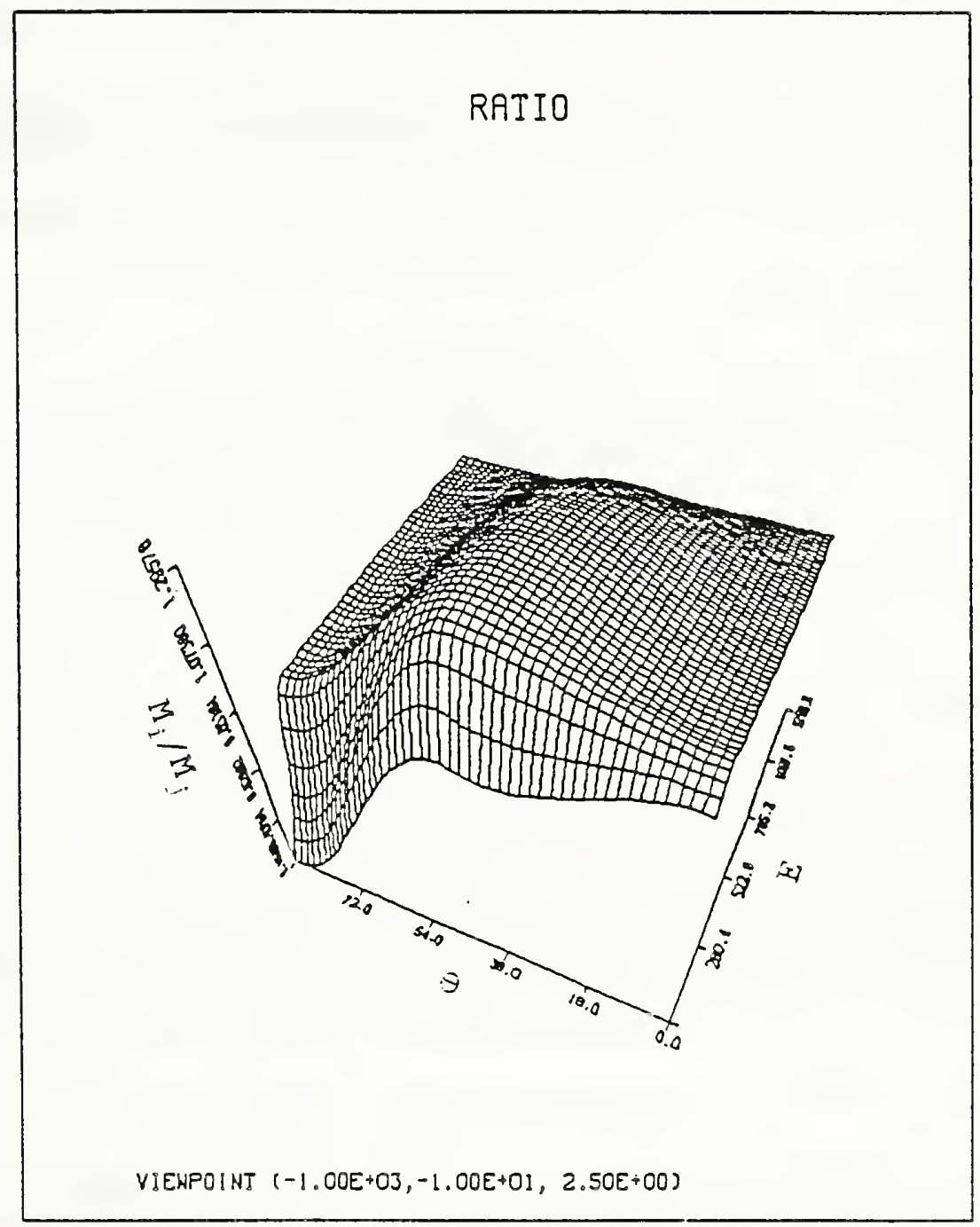

Figure 3. Example of three-dimensional spline of a response-function ratio, $M_{l} / M_{j}[13]$. 
Evaluation of $H$ on the field dosimeters entails:

(a) measuring values of $M$ for the detectors $i$ and $j$ of each field dosimeter, and forming the response ratios, $M_{i} / M_{j}$;

(b) finding the $(E, \theta)$ pair (or, in general, pairs) that correspond to the values of $M_{i} / M_{j}$ obtained in step (d) of section 3.2 , on the calibration surfaces or curves representing $M_{i}(E, \theta) / M_{j}(E, \theta)$ as a function of $E$ and $\theta$;

(c) obtaining the values (or sets of values) of $M_{i} / H$ and $M_{j} / H$ for each set of $(E, \theta)$ pairs on all field dosimeters, with the aid of the calibration data of $M_{i}(E, \theta) / H$ and $M_{j}(E, \theta) /$ $H$, respectively (step (c), sec. 3.2); and

(d) determining the value of $H$ corresponding to each measured value of $M_{i} / M_{j}$ from the calibration relationships between $M_{i}, M_{j}$ and $H$ (step (b) of sec. 3.2).

\subsection{Multiplicity of Results}

Step (b) of the algorithm outlined in section 3.3 generally results in a set of different $(E, \theta)$ pairs, all corresponding to the same measured $M_{i} / M_{j}$ ratio. Their number is likely to increase with the number of calibration energies and angles; but so is the likelihood that they, as a set, reflect more accurately the actual field-irradiation conditions - for which energy spectra and angular distributions may be very different from those used during calibration. Also, it is to be expected that the set of the more reliable results will stem from values of $M$ measured on the detector $i$ or $j$ for which the response functions vary more strongly with $E$ and $\theta$.

\subsection{Assigning Weights to the Resulting Individual $H$ Values}

One then needs to decide on a method for arriving at a unique value of $H$ representing the set of $H$ values obtained in step (d). In first approximation, one may consider taking the algebraic (unit-weight) mean of the $H$ values in each set. This is the simple-minded approach for which results for the more $E$ - and $\theta$-dependent detector were shown in the original presentation. [8] Following is a detailed discussion of the mechanics involved in some of the individual steps of the proposed algorithm, suggesting a more plausible averaging method. The basic idea for this method, which as yet has not been tried, originated with M. Danos [14], and was later elaborated on in discussions with S. M. Seltzer [15].

\subsubsection{Mechanics Involved in Arriving at $(E, \theta)$ Values}

Let us assume that the calibration surfaces representing $M_{i}(E, \theta) / H(E, \theta), M_{j}(E, \theta) /$ $H(E, \theta)$, and $M_{i}(E, \theta) / M_{j}(E, \theta)$ as functions of $E$ and $\theta$ (sec. 3.2) have been fitted numerically, and that coordinates of surface points for equidistant values along the $E$ and $\theta$ axes ("grid points") have been obtained. Let us further assume that the response ratios, $M_{i} / M_{j}$, have been determined on the field dosimeters by step (a) of the algorithm (sec. 3.3). One then is ready for step (b), which entails cutting the calibration surfaces representing $M_{i}(E, \theta) / M_{j}(E, \theta)$ as a function of $E$ and $\theta$ by planes given by

$$
M_{i} / M_{j}=\text { constant }
$$


where $M_{i} / M_{j}$ is one of the ratios derived from the measurements. This step results in a curve of intersection, with points representing the set of $(E, \theta)$ pairs for the particular $M_{i} / M_{j}$ ratio. The subsequent steps (c) and (d) then may be carried out for all the points along the length, $l$, of the curve of intersection, resulting in a set of $H$ values corresponding to these points.

\subsubsection{Averaging the $H$ Values Along the Curve of Intersection}

Let us pick a set of $(E, \theta)$ pairs along the curve of intersection of the plane, $M_{i} / M_{j}=$ constant with the surface representing $M_{i}(E, \theta) / M_{j}(E, \theta)$ as a function of $E$ and $\theta$, that has equidistant spacing of either the $E$ or the $\theta$ values, i.e., that is either of the form

$$
(E, \theta)_{k} \equiv\left(E_{k}, \theta\right)
$$

or of the form

$$
(E, \theta)_{k} \equiv\left(E, \theta_{k}\right)
$$

where $k$ is a running index. Each of these points can be associated with a length, $\Delta l_{k}$, along the curve, where $\Delta_{k}$ may be approximated as

$$
\Delta l_{k} \approx+\sqrt{\left(E_{k+1}-E_{k}\right)^{2}+\left(\theta_{k+1}-\theta_{k}\right)^{2}}
$$

Let us then designate by $H\left(E_{k}, \theta_{k}\right)$ the value of $H$ obtained in step (d) for the length increment, $\Delta l_{k}$, of the curve of intersection. A plausible value, $H_{p}$ representing the set of $H\left(E_{k}, \theta_{k}\right)$ values along the line $l$, would be the weighted average given by

$$
H_{\mathrm{p}}=\sum_{k=1}^{n} H\left(E_{k}, \theta_{k}\right) \Delta l_{k} / \sum_{k=1}^{n} \Delta l_{k}
$$

Alternatively, one might try to obtain similarly weighted averages of the values of $E_{k}$ and $\theta_{k}$ along the curve of intersection obtained in step (b), and then proceed with steps (c) and (d) only for the one $\left(E_{\mathrm{p}}, \theta_{\mathrm{p}}\right)$ pair.

\section{Accuracy of the Method}

At this stage, it is difficult to predict how accurate the results will be that one can obtain with the described algorithm. Among other things, accuracy will depend on such factors as the shape of the response functions and response-function ratios of a particular type of dosimeter, and how well these functions are known-i.e., on how many calibration points are available. Once the numerical procedures are worked out, the algorithm should be tested on a type of dosimeter that fulfills the criteria given in section 3 , for which data are available for a sufficient number of radiation energies and angles of radiation incidence.

The test could be carried out with photons, neutrons or beta particles, or a mixture of them. In the latter case, the assumption could be made that the discrimination between the 
types of radiation has been carried out successfully beforehand. Also, initially, the test could be run as if the calibration dosimeters were identical with the field dosimeters for which irradiation levels, energies and angles are unknown. But this leads to an inherent advantage regarding the compatibility of calibration and field conditions. Therefore, the test must be extended also to "field dosimeters" irradiated at energies and angles different from those used during calibration.

\section{Acknowledgments}

The author wishes to thank R. F. Boisvert of the NIST Center for Applied Mathematics for having adapted his computer program to producing three-dimensional splines of dosimeter response functions and of their ratios, samples of which are shown in the figures. She also acknowledges with thanks the helpful suggestions made by M. Danos, C. Eisenhauer and S. F. Seltzer of the NIST Center for Radiation Research.

\section{References}

[1] Determination of dose equivalents resulting from external radiation sources. Report 39; International Commission on Radiological Units; Bethesda, MD; 1985.

[2] Marshall, T. O.; Bartlett, D. T.; and Burgess, P. H. Current and furure instrument and dosemeter designs to measure the new ICRU radiation quantities. J. Soc. Radiol. Prot. 7(3): 107-118; 1987.

[3] King, C. W. The angular energy response of a personnel dosimeter. 32nd Annual Meeting of the Health Physics Society. Abstracts: S35; 1987.

[4] Radiation Protection Dosimetry 12(2); 1985.

[5] Radiation Protection Dosimetry 28(1-2); 1989.

[6] Sims, C. S. Review of ANSI N.13.11: A status report. Proceedings of the Second Conference on Radiation Protection and Dosimetry. R. E. Swaja and C. S. Sims, editors: ORNL/TM-10971; 1988.

[7] American National Standard for Dosimetry-Personnel Dosimetry-Criteria for Testing. ANSI N.13.11- 1983; American National Standards Institute, Inc.; 24 p.

[8] Ehrlich, M. A method for evaluating air kerma and directional dose equivalent for currently available multi-element dosemeters in radiation protection dosimetry. Rad. Prot. Dosim. 28(1-2): 89-95; 1989.

[9] Ehrlich, M. The use of film badges for personnel monitoring. LAEA Safety Series, No. 8; International Atomic Energy Agency, Vienna; 1962; 80 p.

[10] Jahr, R; Siebert, B. R. L.; and Alberts, W. G. Operational quantities and calibration procedures for individual monitoring. Rad. Prot. Dosim. 28(1-2): 33-36; 1989.

[11] Dennis, J. A. Celestial spheres and mortal men. Rad. Prot. Dosim. 22(3): 147; 1988.

[12] Ehrlich, M. How to overcome the difficulties with the operational dose equivalent quantities. Rad. Prot. Dosim. 29(3): 157-158; 1990.

[13] Boisvert, R. F. NIST Center for Applied Mathematics, private communication; 1988.

[14] Danos, M. NIST Center for Radiation Research, private communication; 1988.

[15] Seltzer, S. M. NIST Center for Radiation Research, private communications; 1988, 1989. 


\section{BIBLIOGRAPHIC DATA SHEET}

4. TITLE ANO SUBTITLE

A Radiation Energy-Angle Algorithm for Use in Personnel Dosimetry

5. AUTHOR(S)

Margarete Ehrlich

6. PEAFORMING ORGANIZATION (IF JOINT OR OTHER THAN NIST, SEE INSTRUCTIONS)

U.S. DEPARTMENT OF COMMERCE

NATIONAL INSTITUTE OF STANDARDS AND TECHNOLOGY

GAITHERSBURG, MO 20899

7. CONTRACT/GRANT NUMBER

9. SPONSORING ORGANIZATION NAME AND COMPLETE ADDRESS (STREET, CITY, STATE, ZIP)

Same as item 6 .

10. SUPPLEMENTARY NOTES

DOCUMENT DESCRIBES A COMPUTER PROGRAM; SF-185, FIPS SOFTWARE SUMMARY, IS ATTACHED.

11. ABSTRACT (A 200-WORD OR LESS FACTUAL SUMMARY OF MOST SIGNIFICANT INFORMATION. IF DOCUMENT INCLUDES A SIGNIFICANT BIBUOGRAPHY OR UTERATURE SURVEY, MENTION IT HERE.)

In an earlier publication, the author described an algorithm for arriving at the value of the individual dose equivalent from the readings of dosimeters having two areas of different energy- and angle-response functions. The algorithm is briefly reviewed here, and suggestions are made for obtaining, by numerical means, a plausible average of all solutions possible for a given type of dosimeter and number of calibration data.

12. KEY WORDS (6 TO 12 ENTRIES; ALPHABETICAL ORDER; CAPITAUZE ONLY PROPER NAMES; AND SEPARATE KEY WORDS BY SEMICOLONS)

algorithm; angular dependence; energy dependence; individual dose equivalent; personnel dosimetry; plausible average of solutions; response functions; response ratios; surfaces in three dimensions; three-dimensional spline fits

FOR OFFICIAL DISTRIBUTION. DO NOT RELEASE TO NATIONAL TECMNICAL INFORMATION SERVICE (NTIS).

ORDER FROM SUPERINTENDENT OF DOCUMENTS, U.S. GOVERNMENT PRINTING OFFICE, WASHINGTON, DC 20402.

ORDER FROM NATIONAL TECHNICAL INFORMATION SERVICE (NTIS), SPAINGFIELD, VA 22161.

14. NUMBER OF PRINTED PAGES

$$
14
$$

15. PAICE 

